\title{
Machine Learning Models to Classify Molecules as Cancer Kinase Inhibitors or Non-Inhibitors
}

\author{
Mihir Putcha \\ Adlai E. Stevenson High School, mihir.putcha@gmail.com
}

\begin{abstract}
Cancer is known to be a leading cause of death worldwide, accounting for nearly 10 million deaths in 2020 alone. Cancer cells reproduce uncontrollably and function aberrantly, sometimes forming malignant or benign tumors that metastasize to other areas of the body. While decades-old cytotoxic chemotherapy that aims to kill tumor cells has had variable success rates, recently, scientists have produced remarkable results with targeted therapies such as cytostatic chemotherapy, which uses anti-cancer drugs to prevent cancer cell proliferation. This project aims to use machine learning models, including 3 dimensional convolutional neural networks to classify checkpoint inhibitors, a type of cytostatic chemotherapy drug, from large pools of molecules. Notably, with reshaped data, the convolutional neural network tested here achieved an AUC of 0.8851 , beating out more sophisticated methods.
\end{abstract}

\section{Background}

Human cells have many different kinases that help control important functions, such as cell signaling, metabolism, division, and survival. Protein kinases are a type of enzyme that are part of a signaling cascade in cells. These kinases transfer a phosphate group onto a target protein that changes the behavior of the protein. They do this by binding ATP and transferring 1 phosphate group to the target protein. The phosphorylated protein and the remaining adenosine diphosphate ( $\mathrm{A}+2$ phosphates) are released. From that point, the phosphorylated protein can then be activated, deactivated, and adjusted to signal other proteins to interact with it. In cancer cells, the phosphorylation of proteins sparked by protein kinases results in the worsening of cancer disorders, allowing cancer cells to proliferate.

Kinase inhibitors, substances that block kinases, are useful in fighting cancers by preventing kinases from phosphorylating proteins and aiding the growth of cancer cells. As a result, they're useful in stemming a variety of cancers, with common kinase inhibitor drugs used in the healthcare industry such as Dasatinib, a tyrosine kinase inhibitor, or PLX5568, an Raf kinase inhibitor. These drugs, also known as checkpoint inhibitors, provide a kind of targeted therapy for patients struggling with cancer.

As new drugs are being tested, it's important that true kinase inhibitor molecules be differentiated from those that are not useful in blocking cancer kinases. This project seeks to classify thousands of different molecules as inhibitors or non-inhibitors by testing several different machine learning models, notably convolutional neural networks for their use of pattern detection.

\subsection{Related Work}

Similar work was performed on an earlier version of the dataset used in this project by N. Balachandar, W. Wang, and C. Liu of Stanford University [1]. Their research involved training separate deep learning models for each of the 8 kinases tested in this project (see section 2.1). Results of their experiment are compared to this project's results in Table 3.

\section{Methodology}

\subsection{Data}

This project uses an updated Cancer Inhibitors dataset from Kaggle [2]. Data was taken from PubChem's chemistry database of molecules and their properties, specifically their physical and chemical properties, identifiers, and chemical structures. Thousands of molecules were gathered from the database that focus on 8 protein kinases. The kinases and total number of molecules for each are outlined in Table 1. The activity record for any given molecule was composed of its Atom Pairs (AP), Morgan Fingerprint (MG), and the Topological Torsions (TT), three different sets of molecular fingerprints generated by RDKit for the PubChem database. 
The three types of fingerprints are each fixed-length bit vectors that encode the identifiable features of a molecule. In summary, Morgan fingerprints are created by incorporating information about circular fragments grown around heavy atoms, atom pairs fingerprints are generated by enumerating the shortest path between all pairs of atoms, and topological torsion fingerprints work by encoding fragments of a molecule for a path of a certain number of bonds. While each fingerprint alone is not unique, the combination of all 3 is a more accurate representation of the individual structure of a molecule.

To accomplish this, each fingerprint set (AP, MG, and TT), which contained 2039 fingerprints, was concatenated, forming $N$ arrays of length 6117 , where $\mathrm{N}$ is the total number of inhibitors and non-inhibitors for that kinase. In turn, these arrays were vertically stacked to create 8 separate features matrices for each of the 8 kinases.

\begin{tabular}{|c|c|}
\hline Protein Kinase & $\begin{array}{c}\text { Total Number of } \\
\text { Molecules }\end{array}$ \\
\hline $\begin{array}{c}\text { Cyclin-dependent kinase 2 } \\
\text { receptor erbB1 }\end{array}$ & 1890 \\
\hline $\begin{array}{c}\text { Glycogen synthase kinase- } \\
\text { beta }\end{array}$ & 6937 \\
\hline $\begin{array}{c}\text { Hepatocyte growth factor } \\
\text { receptor }\end{array}$ & 2879 \\
\hline $\begin{array}{c}\text { MAP kinase p38 alpha } \\
\text { gyrosine-protein kinase } \\
\text { LCK }\end{array}$ & 4670 \\
\hline $\begin{array}{c}\text { Tyrosine-protein kinase } \\
\text { SRC }\end{array}$ & 3587 \\
\hline $\begin{array}{c}\text { Vascular endothelial } \\
\text { frowth receptor 2 }\end{array}$ & 6860 \\
\hline
\end{tabular}

Table 1: Each protein kinase in the dataset and the corresponding total number of inhibitors and noninhibitors

\subsection{Models}

Two types of models were created to classify each molecule as an inhibitor of a protein kinase or a non-

\footnotetext{
${ }^{1}$ Fully connected neural networks will henceforth be referred to as fully connected models (FCM), to avoid confusion with convolutional neural networks.
}

inhibitor. Each model has separate architecture. The configurations of each are outlined in Table 2. The ReLU activation function was not added to the table for the sake of brevity but is present in each convolutional and fully connected layer, save the last layer.

\subsection{Fully Connected Neural Network}

While fully connected models $\left(\mathrm{FCM}^{1}\right)$ lack the ability to process spatial information in this molecular context, they make no assumptions about the structure of the input and thus were a great baseline for this project.

Two FCMs were used in this project that connect every neuron in one layer to every other neuron. In this type of model, also known as a Multi-Layer Perceptron, inputs that enter the consecutive hidden layers are multiplied by weights, or the strength of a connection between two neurons. The final output layer has an activation function to generate the predicted feature. For the FCMs used here, the features matrix for each of the 8 kinases were stacked vertically on top of each other, creating a larger features matrix of size $31200 \times 6117$. Each kinase matrix was stratified separately into 2 submatrices, with train-test splits of $80 / 20$. Consequently, the 8 training submatrices and 8 testing submatrices were vertically stacked to form 2 matrices of size $(24958,6117)$ and $(6242,6117)$ respectively; these matrices were used to train and test the FCMs.

\subsection{Convolutional Neural Networks}

With the aim of finding structural similarities between the molecules, two convolutional neural networks were tested on the dataset. Normally used to recognize patterns in images, CNNs can be useful in identifying patterns between other objects whose structural similarities can correlate to similar properties, namely molecules. CNNs work by taking input images and passing them through a series of convolution layers using neurons (filters) that scan across the receptive field piece by piece. Each image is downsized to create a new feature-filtered image and the process is repeated until an activation function determines the output.

The whole process is done through convolutional, pooling, activation and full connected layers that pass information onto each other. After filters are applied to the image in convolutional layers, pooling layers subsequently reduce the image to smaller dimensions. 
Activation functions introduce non-linearity before the data is fed to the fully connected layer which outputs a one-dimensional feature vector.

While CNNs can be used to analyze 2-dimensional images, this project uses a 3-dimensional CNN to analyze the three-dimensional configuration of the molecules inputted to the model. Thus, the data is transformed to an input shape of 3 dimensions that encourages the model to find numerical similarities between the molecular fingerprints, and therefore structural similarities between the molecules themselves. For the convolutional neural networks, both the training and testing matrices designed for the FCMs were reshaped so that the atom pairs fingerprint lay above the Morgan fingerprint, which in turn lay above the topological torsions fingerprint (Fig. 2, left). This new input shape keeps the 80/20 train-test split while placing individual molecular fingerprints (AP, MG, and TT) parallel to the number of examples to promote the discovery of similar structures by the CNN for each. The final training and testing reshaped matrices were of size $(24958,2039,3)$ and $(6242,2039,3)$ respectively.
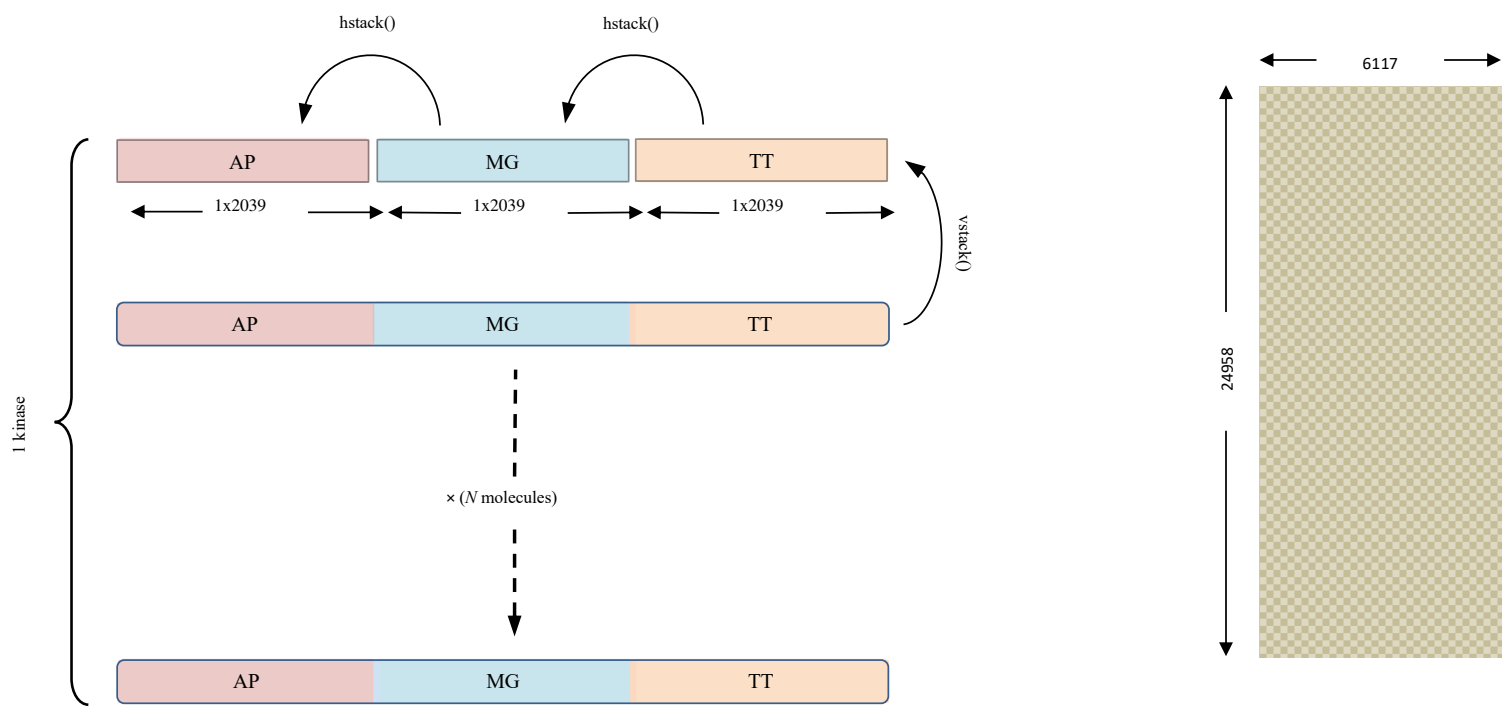

Figure 1: Left: A training/testing features matrix for a single kinase, with molecular fingerprints for thousands of inhibitors and non-inhibitors. Right: The finalized input shape fed into the FCMs.
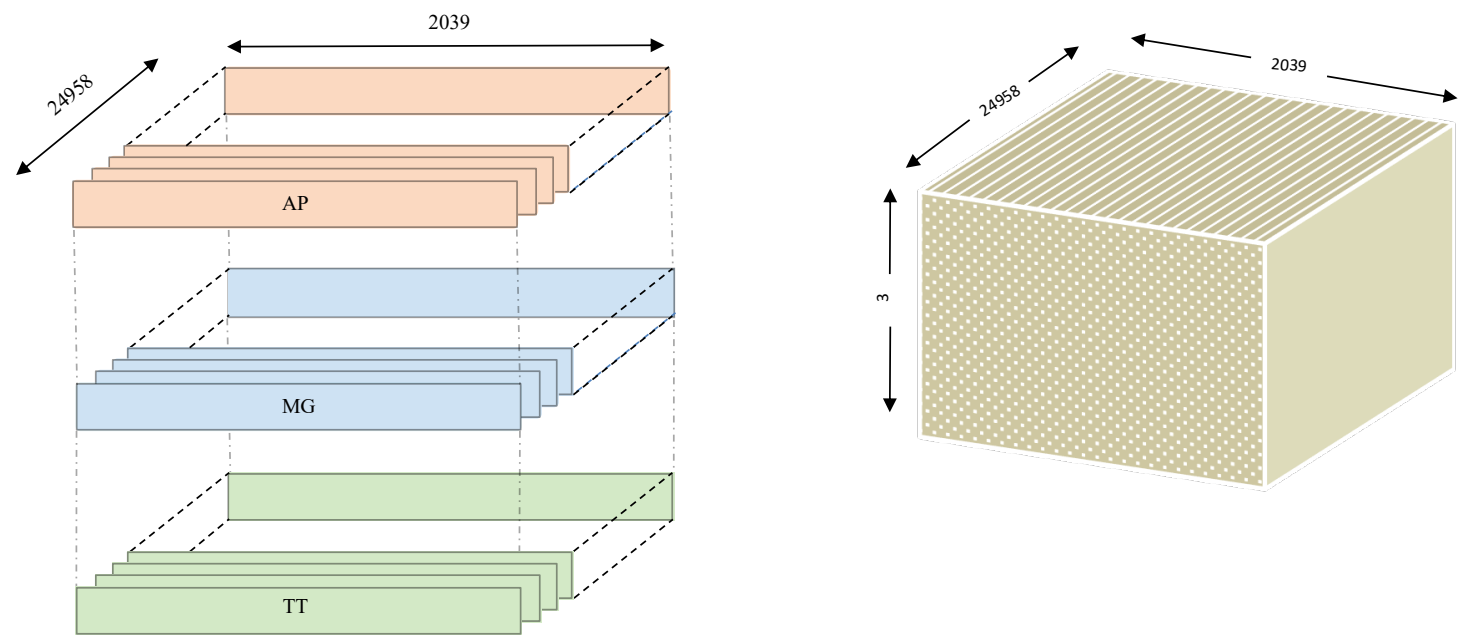

Figure 2: Left: Construction of the reshaped training features matrix fed into the CNNs. Right: The final input shape. 


\begin{tabular}{|c|c|c|c|}
\hline FCM 1 & FCM 2 & CNN 1 & CNN 2 \\
\hline 5 layers & 7 layers & 7 layers & 13 layers \\
\hline FC-1000 & FC-1000 & conv3-64 & conv3-256 \\
\hline FC-100 & FC-500 & maxpool-2 & maxpool-2 \\
\hline FC-100 & FC-500 & conv3-64 & conv3-128 \\
\hline FC-100 & FC-100 & maxpool-2 & conv3-128 \\
\hline FC-1 & FC-100 & conv3-32 & maxpool-2 \\
\hline-------- & FC-100 & FC-100 & conv3-64 \\
\hline------- & FC-1 & FC-1 & conv3-64 \\
\hline-------- & ------- & ------- & maxpool-2 \\
\hline------- & ------- & ------- & conv3-32 \\
\hline------- & ------- & ------- & conv3-32 \\
\hline------- & -------- & ------- & maxpool-2 \\
\hline-------- & ------- & ------- & FC-100 \\
\hline------- & ------- & ------- & FC-1 \\
\hline
\end{tabular}

Table 2: Model architectures. Note that dropout regularization was applied after each fully connected layer, with the dropout ratio set to 0.2 . The last layer activation function for each model was replaced with Sigmoid instead of ReLU.

\section{Results}

The models input the combined features matrix of all the kinase inhibitors before converting the data to 4 output values: accuracy, AUC, recall, and precision.

The classification accuracy is calculated as the number of correct predictions divided by the total number of predictions. While accuracy is a simple and commonly used performance indicator, the classes in this dataset are skewed with a majority of examples of labeled as noninhibitors rather than inhibitors. Because of this, precision is also included as a metric, or the number of correctly predicted positive examples as a fraction of the total number of positive examples. Recall is similar, calculating the percentage of actual positives a model correctly identified. Lastly, the AUC was included to quantify the relationship between precision and recall. Metrics closer to 1.0 indicate better performance in all categories.
Classification metrics were calculated incrementally, by epoch, over varied training periods. These lengths of time were sufficient to determine where training metric values stopped increasing and what their final values were. The training metrics are visualized in graph form in Figure 3. The final testing metrics are listed in Table 3.

\subsection{Training Evaluation}

For accuracy, AUC, and precision, both fully connected models and the first CNN plateaued relatively quickly, with incremental increases in performance afterwards not graphed. On the other hand, the second CNN took a much longer time before beginning to approach an upper bound of 0.9 AUC after at least 8000 seconds for all three metrics. FCM 1 and CNN 1 both achieved high accuracies and AUCs with the CNNs achieving higher precisions. The first CNN showed better accuracy, AUC, and precision than all other models. While the CNN 2 exhibited a slower but steadier growth rate; while CNN 2 could potentially outperform other models with more training time, this is unlikely considering that an upper bound was reached for all metrics by all models.

Interestingly, there was a noticeable recall gap between both fully connected models and the convolutional neural networks. Along with the increased precision of first $\mathrm{CNN}$ and high growth rate of the second $\mathrm{CNN}$, designing an ensemble model would be a reasonable and efficacious idea for future work.

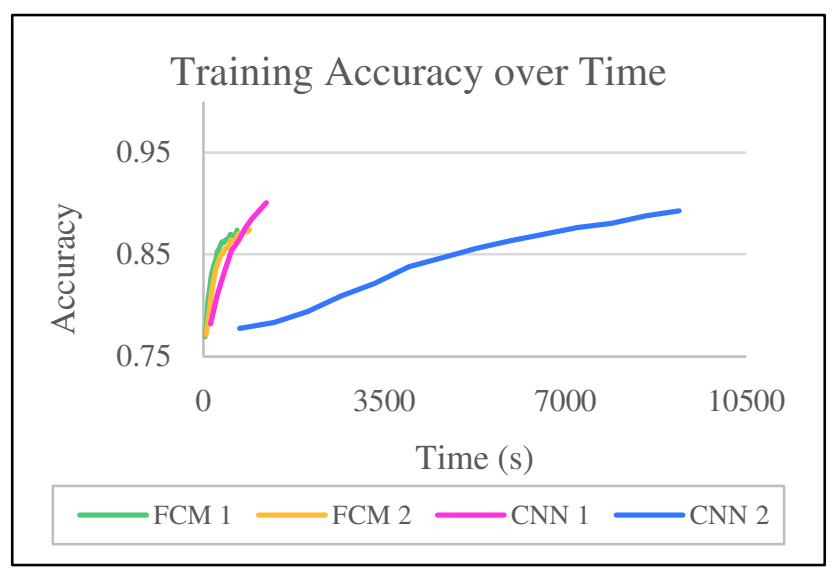

(a) 


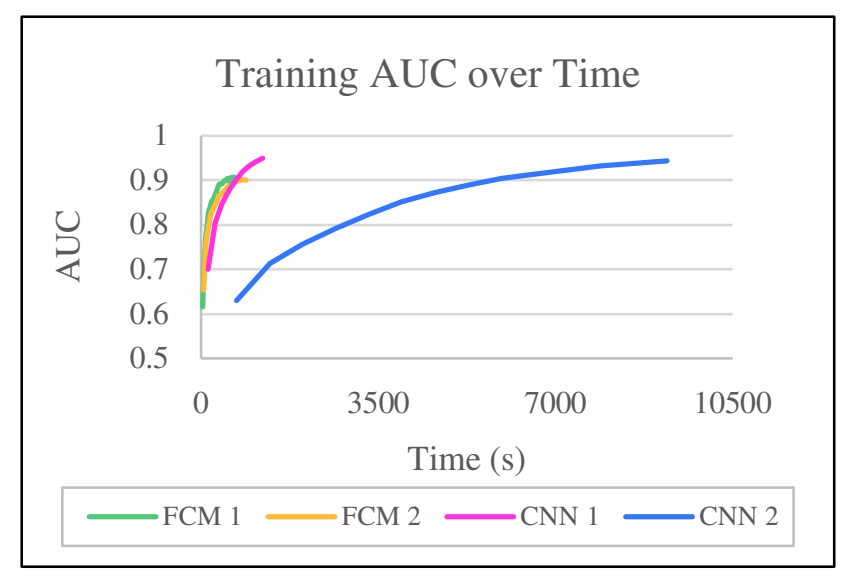

(b)

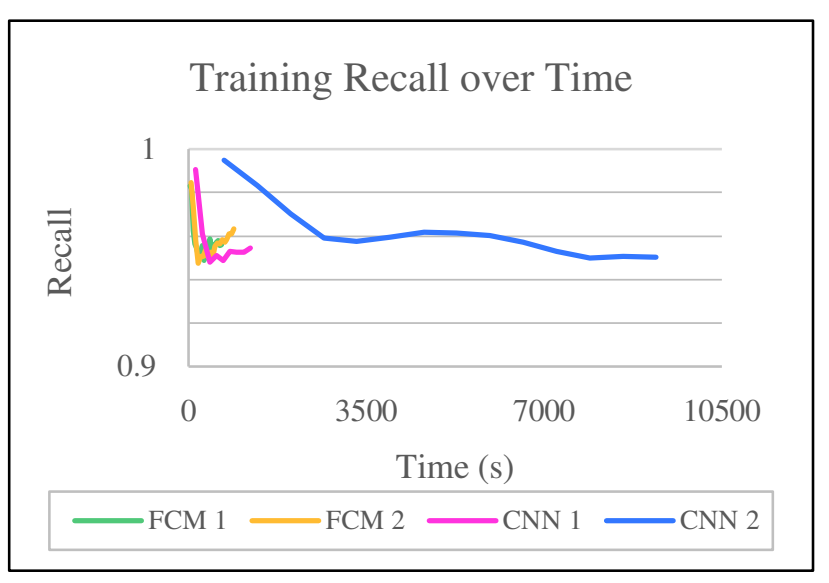

(c)

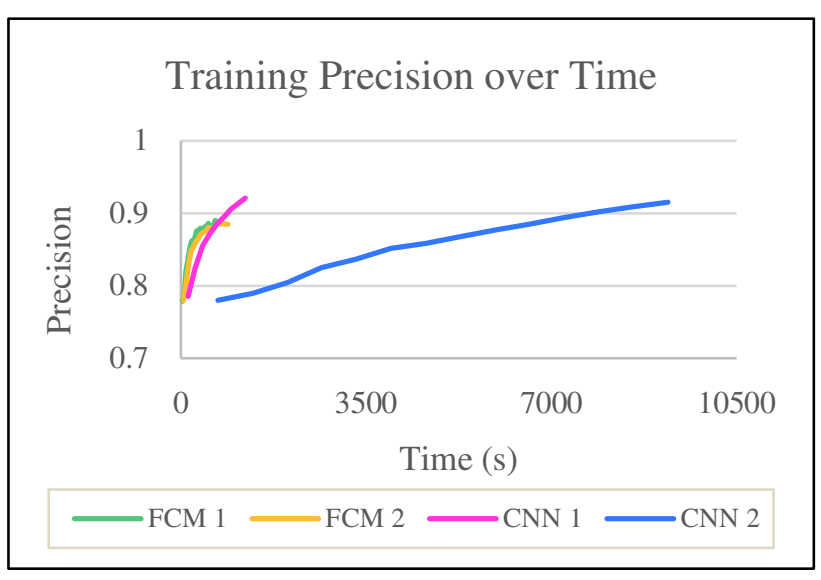

(d)

Figure 3: Training Metrics. Positive slopes indicate increasing model performance.

\footnotetext{
${ }^{2}$ Test AUC was calculated as the average of the best model results from both random/cluster splits in the Stanford research paper [2].
}

\subsection{Testing Evaluation}

\begin{tabular}{|c|c|c|c|c|}
\hline Model & Accuracy & AUC & Recall & Precision \\
\hline FCM 1 & 0.8525 & 0.8676 & $\mathbf{0 . 9 6 0 3}$ & 0.8650 \\
\hline FCM 2 & 0.8515 & 0.8727 & 0.9541 & 0.8681 \\
\hline CNN 1 & $\mathbf{0 . 8 6 3 0}$ & $\mathbf{0 . 8 8 5 1}$ & 0.9378 & $\mathbf{0 . 8 9 1 8}$ \\
\hline CNN 2 & 0.8464 & 0.8614 & 0.9380 & 0.8738 \\
\hline Stanford & ---- & 0.8385 & ---- & ---- \\
\hline RFC & .8606 & .879 & .947 & .877 \\
\hline
\end{tabular}

Table 3: Final testing metrics.

FCM 1 clearly performed best for recall, which is an important metric considering that identifying inhibitors is more important than missing them. Despite this, it was clear CNN 1 performed best for all other metrics including AUC, one of the best indicators of classification model performance for datasets with uneven class distributions, such as this one.

The Random Forest Classifier performed similarly to other models because the amount of data is relatively small and larger models such as the CNN 2 are overfitting the data, thus performing badly on testing sets. From these results, it is reasonable to conclude that neural networks won't be able to reach 0.9 AUC on this traintest split because of the nature of the data, regardless of training time.

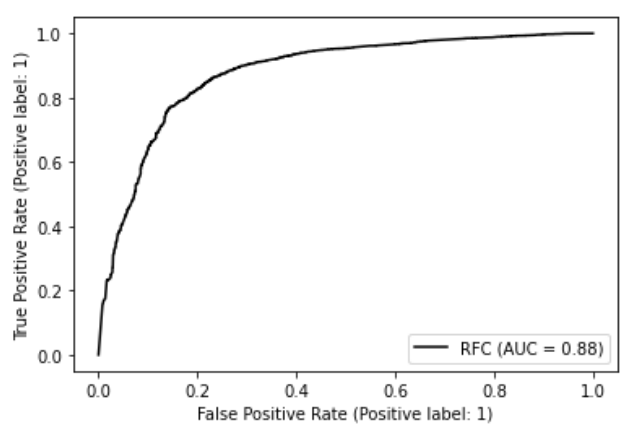

(a) 


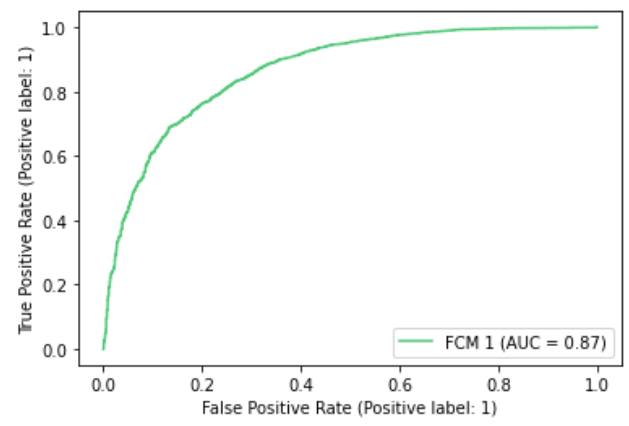

(b)

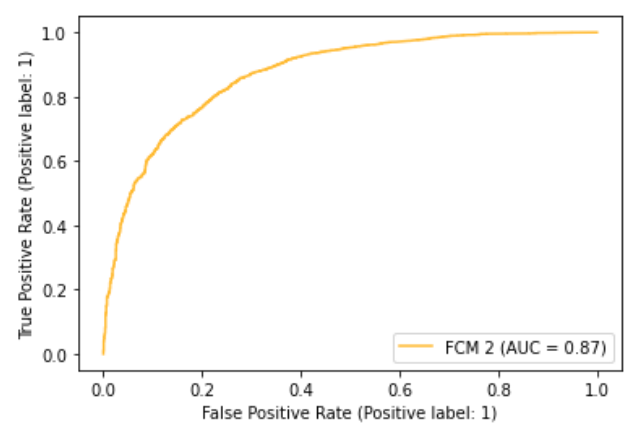

(c)

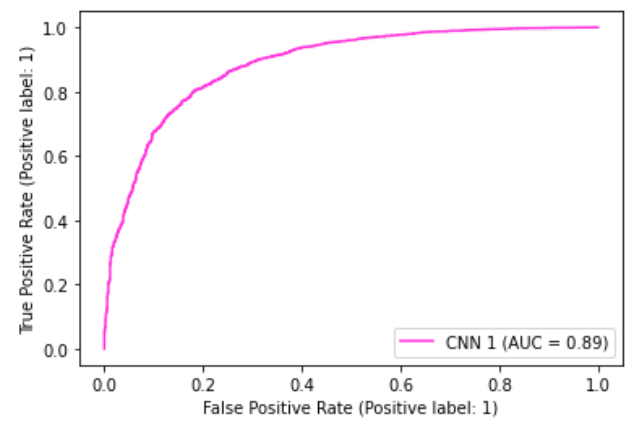

(d)

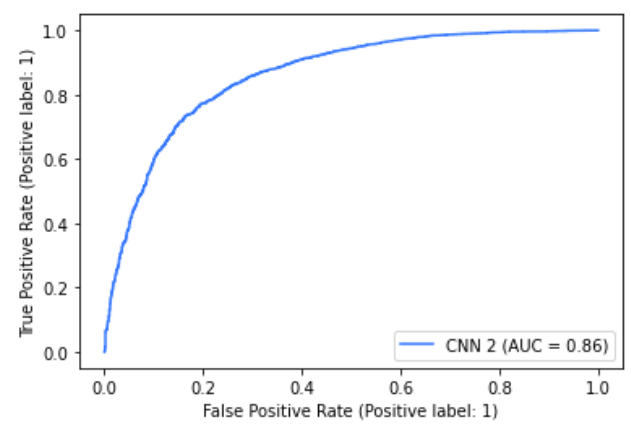

(e)

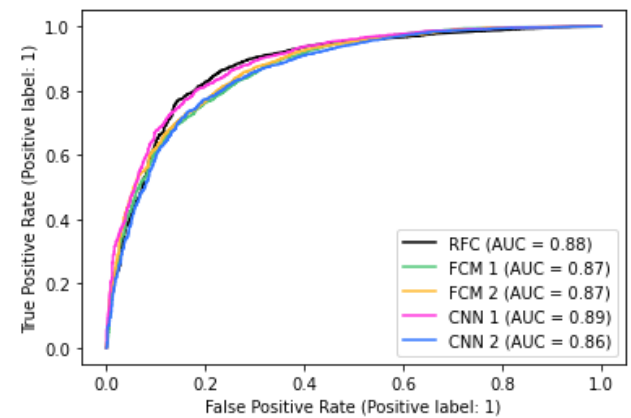

(f)

Figure 4: Test AUCs for each model. Graph (f) models all plots on a single graph.

\section{Conclusion}

Models like these are important in helping identify inhibitors of cancer cells efficiently and effectively. A novel approach to finding an effective classification model was the use of convolutional neural networks on reshaped data to look for structural similarities in 3 dimensions. The data input and architecture of the CNNs were intentionally engineered to take advantage of the molecular structure of kinase inhibitors, which is a likely reason why the CNN performed the best out of all the models presented (in terms of AUC). The results proved that the added complexity of other models does not necessarily yield better results than a well thought out model.

As work like this is completed, new drugs and kinase inhibitors can be immediately marked as potential candidates for cytostatic chemotherapy, accelerating the progression of finding and generating new targeted treatments. Truly, with the help of machine learning models, work and labor involved in this process has reduced 10-fold. Future work on this project could benefit by testing the models upon individual fingerprints and analyzing differences and similarities in results. In addition, while atom pairs, Morgan fingerprints, and topological torsions are excellent candidates for unique molecules, other molecular fingerprints can be utilized as inputs to the model including FP2 and MACCS fingerprints.

\subsection{Acknowledgements}

I would like to thank Zachary-Hervieux Moore from Princeton University for his guidance throughout this project. 


\section{References}

[1] Balachandar, N., Liu, C., \& Wang, W. (2019). Prediction of Small Molecule Kinase Inhibitors for Chemotherapy Using Deep Learning. ArXiv, abs/1907.00329.

[2] K, Xiao. (2019, November). Cancer inhibitors dataset, Version 2. Retrieved October 10, 2021 from https://www.kaggle.com/xiaotawkaggle/inhibitors

\section{Bibliography}

Lavanya V, Mohamed Adil A.A, Neesar Ahmed, Arun K. Rishi, Shazia Jamal (2014) Small molecule inhibitors as emerging cancer therapeutics. Integr Cancer Sci Therap. 1: DOI: 10.15761/ICST.1000109

Kumar M, Nagpal R, Hemalatha R, Verma V, Kumar A, Singh S, Marotta F, Jain S, Yadav H. Targeted cancer therapies: the future of cancer treatment. Acta Biomed. 2012 Dec;83(3):220-33. PMID: 23762999 .

Capecchi, A., Probst, D. \& Reymond, JL. One molecular fingerprint to rule them all: drugs, biomolecules, and the metabolome. $\mathrm{J}$ Cheminform 12, 43 (2020). https://doi.org/10.1186/s13321-02000445-4

Glem, Robert \& Bender, Andreas \& Hasselgren, Catrin \& Carlsson, Lars \& Boyer, Scott \& Smith, James. (2006). Circular fingerprints: Flexible molecular descriptors with applications from physical chemistry to ADME. IDrugs : the investigational drugs journal. 9. 199-204.

Capecchi, Alice, et al. "One Molecular Fingerprint to Rule Them All: Drugs, Biomolecules, and the Metabolome." Journal of Cheminformatics, vol. 12, no. 1, June 2020, p. 43. BioMed Central, https://doi.org/10.1186/s13321-020-00445-4.

Balachandar, N., Liu, C., \& Wang, W. (2019). Prediction of Small Molecule Kinase Inhibitors for Chemotherapy Using Deep Learning. ArXiv, abs/1907.00329.

Syrine Ben Driss, Mahmoud Soua, Rostom Kachouri, Mohamed Akil. A comparison study between MLP and Convolutional Neural Network models for character recognition. SPIE Conference on Real- Time Image and Video Processing, Apr 2017, Anaheim, CA, United States. 10.1117/12.2262589 . hal-01525504

Cancer. (2021, September 21). https://www.who.int/news-room/factsheets/detail/cancer

Eva Kiesler, M. B. (2016, May 10). The Future of Cancer Research: Five Reasons for Optimism | Memorial Sloan Kettering Cancer Center. https://www.mskcc.org/news/future-five-reasons-optimism

National Institute of Mental Health. (n.d.). Immune Checkpoint Inhibitors-National Cancer Institute. Retrieved January 30, 2022, from https://www.cancer.gov/aboutcancer/treatment/types/immunotherapy/checkpoint-inhibitors

National Institute of Mental Health. (2011, February 2). Definition of immune checkpoint inhibitor-NCI Dictionary of Cancer TermsNational Cancer Institute (nciglobal,ncienterprise) [NciAppModulePage].https://www.cancer.gov/publications/dictiona ries/cancer-terms/def/immune-checkpoint-inhibitor
National Institute of Mental Health. (2021, December 6). Targeted Cancer Therapies Fact Sheet-National Cancer Institute (nciglobal,ncienterprise) [CgvArticle]. https://www.cancer.gov/about-cancer/treatment/types/targetedtherapies/targeted-therapies-fact-sheet

OpenEye Scientific Software. (2021, December 3). Fingerprint Generation-Toolkits-Python. https://docs.eyesopen.com/toolkits/python/graphsimtk/fingerprint.ht $\mathrm{ml}$

Kourou, K., Exarchos, T. P., Exarchos, K. P., Karamouzis, M. V., \& Fotiadis, D. I. (2015). Machine learning applications in cancer prognosis and prediction. Computational and Structural Biotechnology Journal, 13, 8-17. https://doi.org/10.1016/j.csbj.2014.11.005

Gu, J., Wang, Z., Kuen, J., Ma, L., Shahroudy, A., Shuai, B., Liu, T., Wang, X., Wang, G., Cai, J., \& Chen, T. (2018). Recent advances in convolutional neural networks. Pattern Recognition, 77, 354-377. https://doi.org/10.1016/j.patcog.2017.10.013

Kobayashi K, Bolatkan A, Shiina S, Hamamoto R. Fully-Connected Neural Networks with Reduced Parameterization for Predicting Histological Types of Lung Cancer from Somatic Mutations. Biomolecules. 2020 Aug 28;10(9):1249. doi: 10.3390/biom10091249. PMID: 32872133; PMCID: PMC7563438.

Emmert-Streib, F., Yang, Z., Feng, H., Tripathi, S., Dehmer, M. (2020). An Introductory Review of Deep Learning for Prediction Models With Big Data. Frontiers in Artifical Intelligence. https://www.frontiersin.org/articles/10.3389/frai.2020.00004/full

Dietterich T.G. (2000) Ensemble Methods in Machine Learning. In: Multiple Classifier Systems. MCS 2000. Lecture Notes in Computer Science, vol 1857. Springer, Berlin, Heidelberg. https://doi.org/10.1007/3-540-45014-9_1

Zhou,J.;Gandomi,A.H.; Chen, F.; Holzinger, A. Evaluating the Quality of Machine Learning Explanations: A Survey on Methods and Metrics. Electronics 2021, 10, 593. https://doi.org/10.3390/ electronics 10050593

Cross-Participant EEG-Based Assessment of Cognitive Workload Using Multi-Path Convolutional Recurrent Neural Networks - Scientific Figure on ResearchGate. Available from: https://www.researchgate.net/figure/Input-shapes-for-each-networktype-are-illustrated-a-The-CNN-input-shape-wasexpanded_fig1_324801331

Kannaiyan, R., \& Mahadevan, D. (2018). A comprehensive review of protein kinase inhibitors for cancer therapy. Expert review of anticancer therapy, 18(12), 1249-1270. https://doi.org/10.1080/14737140.2018.1527688

Anttila JV, Shubin M, Cairns J, Borse F, Guo Q, Mononen T, et al. (2019) Contrasting the impact of cytotoxic and cytostatic drug therapies on tumour progression. PLoS Comput Biol 15(11): e1007493. https://doi.org/10.1371/journal.pcbi.1007493

Pucci, C., Martinelli, C., \& Ciofani, G. (2019). Innovative approaches for cancer treatment: current perspectives and new challenges. Ecancermedicalscience, 13, 961. https://doi.org/10.3332/ecancer.2019.961 